\title{
Supplementary methods for Medical Food Assessment Using a Smartphone App With Continuous Glucose Monitoring Sensors: Proof-of-Concept Study
}

\section{Prepossessing and Data Normalization}

The Libre sensors used in this study had a maximum lifespan of 14-days with a subset of these limited to 10-days. The sensors produce unreliable glucose concentration data for the first 6-24 hours of their use. CGM are less accurate in the first (and sometimes second) day; this inaccuracy has been attributed to the host-immune response to the application of the sensor (see below) Figure 1). Sensors are also likely to have reduced performance in the last day probably due to collagen formation around the sensor (see paragraph Accuracy in [1]). For these reasons, the CGM data from the first (and sometimes second) and last day of the sensors were not used in most statistical analyses.

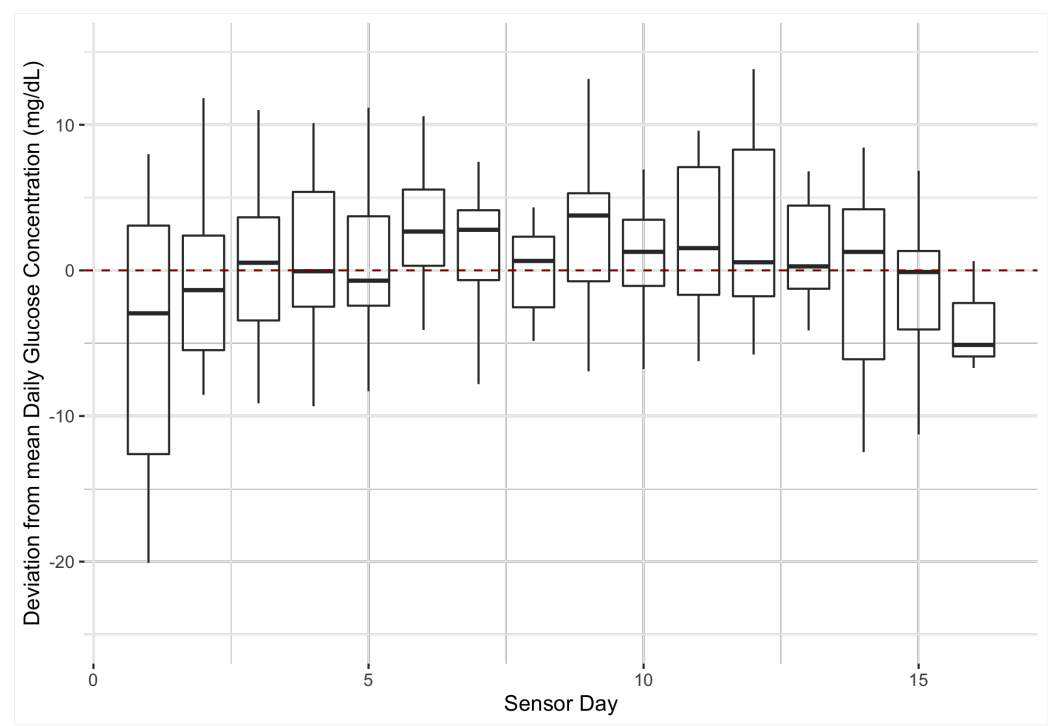

Figure 1: Sensor age. First and last day of a sensor were removed from the analysis because CGM underestimate glucose concentration during those days. One hypothesis is that the sensors cause local inflammation during the first (and sometimes second day), and that collagen starts forming around the sensor during the last day.

\section{Product}

Four out of the five synbiotic strains (Akkermansia muciniphila, Eubacterium hallii, Clostridium beijerinckii and Clostridium butyricum) were manufactured at Pendulum Therapeutics according to current Good Manufacturing Practices (cGMP) for food products in an FDA registered food manufacturing facility. Those strains were each grown in 2000-litre anaerobic batch cultures using media free of animal products and then harvested mid-log phase by centrifugation. Afterwards, individual strains were mixed to obtain the final formulation, which was then put into the capsules. The complete characterization of these strains along with data from preclinical rat toxicity and human-safety pilot studies were combined into a Generally Recognized as Safe (GRAS) dossier 
which was reviewed and accepted by an independent third party (Ramboll Environ, Arlington, $\mathrm{VA})$.

The four strains described above were also mixed with the commercially available strain B. infantis, purchased from Nutraceutix (Redmond, WA) as lyophilized powder. Combined study product was encapsulated in acid-resistant opaque capsules and stored at $4^{\circ} \mathrm{C}$ until use. The formulation contained between $9.0 \times 10^{8}$ and $1.6 \times 10^{1} 0$ Active Fluorescent Units (AFU), a quantitative measure of viable-cell count, of each of the specified bacteria. The placebo study product was composed of colloidal silicon dioxide (CSD). All participants were instructed to consume 3 capsules of their assigned study product twice daily prior to consuming their morning and evening meals.

\section{$3 \quad$ Fecal Microbiome Analyses}

All but one participant agreed to provide stool samples. Whole stool was collected by participants using a Commode Specimen Collection System (Biomedical Polymers, Sterling, MA). Upon collection, subjects were instructed to immediately freeze (home freezer, approximately $-20{ }^{\circ} \mathrm{C}$ ) their stool samples within a sponsor-provided thermally-insulated container. After returning to Pendulum Therapeutics, samples were kept for long-term storage at $-80{ }^{\circ} \mathrm{C}$. Stool samples were first thawed for homogenization, which consisted of: (1) suspension in a 2:1 ratio $(\mathrm{m} / \mathrm{m})$ of 50 $\mathrm{mM}$ Tris $\mathrm{HCl}$ buffer ( $\mathrm{pH} 8.0$ ) with $5 \mathrm{mM}$ EDTA, (2) homogenization for five minutes in a paddle stomacher (Stomacher 3500, Seward, UK), (3) filtration through a coarse $280 \mu \mathrm{m}$ mesh, and (4) preparation of $1 \mathrm{~mL}$ aliquots subsequently refrozen and maintained at $-80{ }^{\circ} \mathrm{C}$.

\section{Measuring anthropometric characteristics}

Weight (W) was measured with Etekcity Stainless Steel Digital Body Weight Bathroom Scale, StepOn Technology. Blood Pressure (BP) and Heart Rate (HR) were measured with iProvèn BPM2244B. O2 levels measured with Santamedical Generation 2 SM-165 Fingertip Pulse Oximeter.

\section{Power calculation}

We want to estimate the sample size of a potential similar future study. In the context of using a one sided t-test, we want to test if the mean $\triangle \triangle A U C$ is negative. The Null and alternative hypotheses are

$$
\begin{array}{ll}
H 0: & \Delta \triangle A U C<0 \\
H 1: & \Delta \triangle A U C \geq 0
\end{array}
$$

Then, formulas to compute sample size $n$ and power $1-\beta$ are

$$
\begin{gathered}
n=\left(\sigma \frac{z_{1-\alpha}+z_{1-\beta}}{\Delta \Delta A U C}\right)^{2} \\
1-\beta=\phi\left(z-z_{1-\alpha}\right)+\phi\left(-z-z_{1-\alpha}\right)
\end{gathered}
$$

where

$$
z=\frac{\Delta \Delta A U C}{\frac{\sigma}{\sqrt{n}}} .
$$

With $\triangle \triangle A U C=1550$ and $\sigma=1568$, if we take $\alpha=0.05,1-\beta=0.9$ (power), the estimated sample size is 37 .

\section{Peak detection}

Consider a set $\mathrm{X}$ of $\mathrm{T}$ equally spaced points $\left\{x_{1}, x_{2}, \ldots, x_{T}\right\}$. We want to find the peaks in this time series. 


\subsection{Finding peak tops}

The peak detection algorithm follows [2]. Two parameters are user-defined: $\mathrm{k}$, which determines how wide the neighborhood around the peaks is, and $\mathrm{h}$, which determines how high peaks have to get. In particular, a smaller $\mathrm{h}$ value lead to more peaks being selected but since those are later filtered, the algorithm is relatively insensitive to the choice of h. We pick $k=7$ and $h=1.5$, based on visual results.

- For each point $x_{i}$, compute $S\left(x_{i}, k\right)=\frac{\max \left(x_{i}-x_{i-1}, x_{i}-x_{i-2}, \ldots, x_{i}-x_{i-k}\right)+\max \left(x_{i}-x_{i+1}, x_{i}-x_{i+2}, \ldots, x_{i}-x_{i+k}\right)}{2}$.

- Define peaks as points where $S\left(x_{j}\right)-\overline{S\left(x_{i}\right)}>h * s d_{i}\left(S\left(x_{i}\right)\right)$.

- If two peaks are 30min or less apart, only keep the one with the highest $\mathrm{S}$ value.

\subsection{Extending peaks}

\section{Start of the peak}

Define the neighborhood Starting from the top of the peak $i$, we extend the neighborhood to the points before, stopping if the end of the previous peak is encountered, the rate is smaller than -5 , the rate is negative for the second time or $\mathrm{k}$ points have been added. We name $\mathrm{K}$ the set of indexes of the left-neighbors of the peak $i$.

Find the start of the peak We define $j_{\text {start }}=\operatorname{argmin}_{j \in K} x_{j}$. If $j_{\text {start }} \neq \min (K)$, then the peak start at $x_{j_{\text {start }}}$. Else, the peak is extended on the left until $j=1$, we encounter the end of the previous peak or the rate decreases. The last point reached is the start of the peak $i$.

\section{End of the peak}

Define the neighborhood Starting from the top of the peak $i$ and we extend the neighborhood to the points after, stopping if we encounter the top of the next peak, the rate is larger than +5 , the rate is positive for the second time or we have added $\mathrm{k}$ points. We name $\mathrm{K}$ the set of indexes of the right-neighbors of the peak $i$

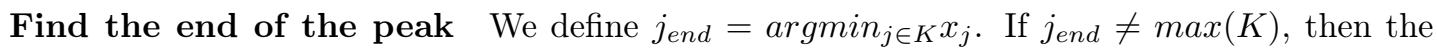
peak ends at $x_{j_{\text {start }}}$. Else, we extend the peak on the right until $j=T$, we encounter the end of the previous peak or the rate increases. The point where we stop is the end of the peak $i$.

\subsection{Merging peaks}

We define a threshold thresh. In practice, we pick thresh $=20$. The threshold is the minimum height of a peak, but also informs the merging criterion.

Let's consider two consecutive peaks $i_{1}$ and $i_{2}$. If $i_{2}-i_{1} \leq k$, that is the two peaks are less than $\mathrm{k}$ points apart, and $\min \left(x_{i_{1}}, x_{i_{2}}\right)-\min _{i_{1}<j<i_{2}}\left(x_{j}\right)<\frac{\text { thresh }}{2}$, that is the glucose drops by less than $\frac{\text { thresh }}{2}$ between the two peak tops, then the peaks were merged. The new peak starts at the start of peak $i_{1}$, ends at the end of peak $i_{2}$, and peaks at $i_{\text {top }}=\operatorname{argmax}_{i \in\left\{i_{1}, i_{2}\right\}} x_{i}$.

\subsection{Filter peaks}

A peak $i$ is filtered out if $x_{\text {top }}^{(i)}-\min \left(x_{\text {start }}^{(i)}, x_{\text {end }}^{(i)}\right)<$ thresh 


\section{References}

[1] Daniel DeSalvo and Bruce Buckingham. Continuous glucose monitoring: current use and future directions. Current diabetes reports, 13(5):657-62, oct 2013. ISSN 1539-0829. doi: 10.1007/s11892-013-0398-4. URL http://www.ncbi.nlm.nih.gov/pubmed/23943230http://www . pubmedcentral.nih.gov/articlerender.fcgi?artid=PMC5164922.

[2] Girish Palshikar. Simple Algorithms for Peak Detection in Time-Series Social Media Analysis View project Human Safety View project Simple Algorithms for Peak Detection in Time-Series Simple Algorithms for Peak Detection in Time-Series, 2009. URL https://www.researchgate.net/publication/ 228853276. 